\title{
A singular Gierer-Meinhardt system of elliptic equations
}

\author{
by \\ Y.S. CHOI ${ }^{1}$, P.J. McKENNA \\ Department of Mathematics, University of Connecticut, Storrs, CT 06269, USA \\ Manuscript received 14 September 1999, revised 6 December 1999
}

ABSTRACT. - We establish the existence of solutions to a singular non-quasimonotone system of equations. Such equations are a special case of the Gierer-Meinhardt equations. In the one dimensional case, the uniqueness result is also proved.

(C) 2000 L'Association Publications de l'Institut Henri Poincaré. Published by Elsevier B.V. All rights reserved

Key words: Singular, Gierer-Meinhardt, Elliptic system, Non-quasimonotone

AMS classification: Primary 35J25

RÉSUMÉ. - Nous établissons içi l'existence des solutions d'un système d'équations singulières non-quasimonotones. Ces équations forment un cas spécial des équations de Gierer-Meinhardt. En dimension un, nous demontrons aussi l'unicité des solutions.

(C) 2000 L'Association Publications de l'Institut Henri Poincaré. Published by Elsevier B.V. All rights reserved

\section{INTRODUCTION}

Singular elliptic boundary value problems for a single equation have been widely studied in the past several decades. While starting out as the

\footnotetext{
${ }^{1}$ Research partially supported by NIH grant 1P41-RR13186-01.
} 
study of ordinary differential equations in [19,20], it rapidly progressed to the study of elliptic nonlinear boundary value problems, see [5,9]. Presently, many of the earlier results are being generalized to different operators, often quasilinear and anisotropic, as these are the type that often occur in applications such as fluid dynamics $[1,3,4,2]$

Although there is a substantial literature on systems of elliptic partial differential equations $[17,6,18]$, to our knowledge, there has been no similar study of results for systems of singular elliptic problems even though they arise naturally in applications.

For example, the system

$$
\begin{aligned}
& u_{t}=d_{1} \Delta u-u+\frac{u^{p}}{v^{q}}, \\
& v_{t}=d_{2} \Delta v-v+\frac{u^{r}}{v^{s}},
\end{aligned}
$$

usually with Neumann boundary conditions, often occurs in the study of morphogenesis on experiments on hydra, an animal of a few millimeters in length, where they are known as the Gierer-Meinhardt equations [13].

With similar interaction terms, these type also occur in certain models of predator-prey interactions [21].

The steady states of (1.1) will be the object of this paper. Often the case that is studied is when the $d_{1}$ term is small and the $d_{2}$ term is large. In this case, the study of solutions of the system can be approximated by a single elliptic equation $[12,7]$.

Of course, with Neumann boundary conditions, one usually studies situations in which the solutions remain positive, so that in some sense the equations do not become singular.

In this paper, we begin the study of steady states of the system with Dirichlet boundary conditions. This is the natural extension of the aforementioned work on the single equation. Specifically, we shall prove two theorems, one an existence theorem for the system for a special choice of the exponents $p, q, r, s$, and the other a uniqueness theorem under the additional assumption that the number of spatial dimensions is one, i.e., we have a singular system of ordinary differential equations.

While there is a good deal of literature on nonlinear elliptic systems, there seems to be little work on this type of singular system, especially if the system is not quasimonotone.

First some preliminaries: our notation will be standard. The norms $\|u\|_{p},\|u\|_{\infty}$ denote the usual $L^{p}$ and $L^{\infty}$ norms on function $u$ on the 
region $\Omega$ in $R^{n}$, respectively. The Sobolev norm $\|u\|_{W^{2, p}}$ will denote its $W^{2, p}$ norm, and $\|u\|_{H^{1}} \equiv\left(\|\nabla u\|_{2}^{2}+\|u\|_{2}^{2}\right)^{1 / 2}$ is its $H^{1}$ norm.

\section{MAIN RESULT}

Let $\Omega \subset \mathbf{R}^{N}$ be a bounded domain with $C^{2+\gamma}$ boundary, where $\gamma \in$ $(0,1)$. We consider positive solutions to the singular system of elliptic equations:

$$
\begin{gathered}
\Delta u-u+\frac{u}{v}=0, \\
\Delta v-\alpha v+\frac{u}{v}=0,
\end{gathered}
$$

with zero Dirichlet boundary conditions:

$$
\left.u\right|_{\partial \Omega}=\left.v\right|_{\partial \Omega}=0
$$

where $\alpha$ is a given positive constant. We note that the system is not quasi-monotone, making direct construction of comparison functions impossible.

THEOREM 1. - Let $\Omega \subset \mathbf{R}^{N}$ be a bounded domain with $C^{2+\gamma}$ boundary, where $\gamma \in(0,1)$. There exist positive solutions $(u, v)$ in $C^{2}(\Omega) \cap$ $C^{1}(\bar{\Omega})$ to Eqs. (2.2) with boundary conditions (2.3). In the one dimensional domain case, the solutions are unique. Moreover such unique solutions $u$ and $v$ are symmetric about the mid point of the one dimensional domain.

Remark 1. - The equations

$$
\begin{array}{r}
\Delta u-u+\frac{u}{v}=0, \\
d \Delta v-\alpha v+\beta \frac{u}{v}=0,
\end{array}
$$

with positive constants $d, \alpha$, and $\beta$ can be reduced to our case. By dividing the second equation by $d$, we can, without loss of generality, assume $d=1$. Now with a new variable $U \equiv \beta u$, we obtain Eqs. (2.2) in $U$ and $v$.

Remark 2. - In the one dimensional case, we can by translation assume the domain is $[-L, L]$ for some $L>0$. Once we establish the existence and the uniqueness of solutions, the symmetry claim in the above theorem is automatic. Since if not, then $U(x)=u(-x)$ and 
$V(x)=v(-x)$ will constitute another solutions. This contradicts the uniqueness result.

Remark 3. - In the proof of this theorem, all the constructions involving functions in $C^{2}(\bar{\Omega})$ (for example, $w$ in Eq. (3.1)) can be replaced by functions in $C^{2}(\Omega) \cap C^{1}(\bar{\Omega})$ only. With $C^{1,1}$ domain, such regularity can be achieved by the $L^{p}$ theory. Hence the theorem is still valid for $C^{1,1}$ domain.

To prove the existence of solutions, we have to divide into three cases: $\alpha<1, \alpha=1$, and $\alpha>1$. First we start with the trivial case $\alpha=1$. The other two cases will be investigated in later sections.

THEOREM 2. - Let $\Omega$ be a bounded domain with $C^{2+\gamma}$ boundary, where $\gamma \in(0,1)$. If $\alpha=1$, then there exists a unique solution $(u, v)$ in $C^{2}(\bar{\Omega})$.

Proof. - Simple algebraic manipulation on Eqs. (2.2) gives the unique solution $u=v=w$, where $w$ satisfies $\Delta w-w+1=0$ with zero Dirichlet boundary conditions. It is noted that the Schauder's estimate on linear elliptic equations ensures that $w \in C^{2}(\bar{\Omega})$.

Throughout this paper, we let $\lambda_{1}>0$ and $\varphi>0$ be the first eigenvalue and the corresponding eigenfunction satisfying

$$
\Delta \varphi+\lambda_{1} \varphi=0,\left.\quad \varphi\right|_{\partial \Omega}=0,
$$

with the normalization $\max _{\bar{\Omega}} \varphi=1$.

\section{EXISTENCE OF SOLUTIONS WHEN $\alpha<1$}

In this section, we study the existence of solutions as stated in Theorem 1 when $\alpha<1$, by using Schauder's fixed point theorem. We can construct an upper bound function (hence a pointwise bound), but to ensure that a positive solution exists, we need to construct a tricky integral lower bound (see the definition of the set $S$ below).

First, we define $w$ to be the solution satisfying

$$
\Delta w-w+1=0,\left.\quad w\right|_{\partial \Omega}=0 .
$$

Hence $w \in C^{2+\gamma}(\bar{\Omega})$. The maximum principle ensures that $w>0$ in $\Omega$.

With $w$ well defined, let $\tilde{w}$ be the solution of the linear equations:

$$
\Delta \tilde{w}-\alpha \tilde{w}=-w,\left.\quad \tilde{w}\right|_{\partial \Omega}=0 .
$$


Again $\tilde{w} \in C^{2+\gamma}(\bar{\Omega})$, and $\tilde{w}>0$ by the maximum principle.

Many apriori estimates for this type of linear equations exist. The results that we will need are summarized as follows.

LEMMA 1. - Let $\beta \geqslant 0$. If

$$
\Delta \hat{z}-\beta \hat{z}=-f,\left.\quad \hat{z}\right|_{\partial \Omega}=0,
$$

then there exists a constant $k_{\beta}>0$ such that $\|\hat{z}\|_{C^{1}} \leqslant k_{\beta}\|f\|_{\infty}$.

Proof. - This is a consequence of $L^{p}$ theory on linear elliptic equations with $p>N$, followed by the Sobolev's imbedding.

When $\alpha<1$, it is easier to study an equivalent system to (2.2). Let $z \equiv v-u$. Then Eqs. (2.2) can be converted into:

$$
\begin{aligned}
\Delta z-\alpha z & =-(1-\alpha) u, \\
\Delta u-u+\frac{u}{u+z} & =0,
\end{aligned}
$$

subject to zero Dirichlet boundary conditions for both $u$ and $z$. We note that this system is still not quasi-monotone if we look for positive solutions for $u$ and $z$.

THEOREM 3. - Let $\Omega$ be a bounded domain with $C^{2+\gamma}$ boundary. If $0<\alpha<1$, then there exist positive solutions $(u, v)$ in $C^{2}(\Omega) \cap C^{1}(\bar{\Omega})$.

Proof. - It suffices to establish existence of positive solutions $(u, z)$ for Eqs. (3.3) with zero Dirichlet boundary conditions on both $u$ and $z$. Then $v=u+z$ will also be positive.

Let $k_{1}$ and $k_{\alpha}$ be the constants in Lemma 1 , when $\beta$ is 1 and $\alpha$, respectively. Define $A \equiv \max \left\{k_{1}, k_{\alpha}\right\}\left(1+\|w\|_{\infty}\right)$, and $M \equiv(1+$ $\left.\lambda_{1}\right)\left(\|w\|_{\infty}+\|\tilde{w}\|_{\infty}\right)$. Let $\delta>0$, to be determined later, and

$$
\begin{array}{r}
S \equiv\left\{(u, z) \in C^{1}(\bar{\Omega}) \times C^{1}(\bar{\Omega}): 0 \leqslant u \leqslant w, 0 \leqslant z \leqslant \tilde{w},\left.u\right|_{\partial \Omega}=\left.z\right|_{\partial \Omega}=0,\right. \\
\left.\int_{\Omega} u \varphi \geqslant \delta, \int_{\Omega} z \varphi \geqslant \frac{1-\alpha}{\alpha+\lambda_{1}} \delta,\|u\|_{C^{1}} \leqslant A,\|z\|_{C^{1}} \leqslant A\right\} .
\end{array}
$$

When $\delta$ is small, $S$ is non-empty. Moreover, $S$ is closed, bounded, and convex.

Define the map $T$ such that for all $(u, z) \in S,(T u, T z)$ solve the linear system of equations: 


$$
\begin{aligned}
\Delta T z-\alpha T z & =-(1-\alpha) u, \\
\Delta T u-T u+\frac{u}{u+T z} & =0,
\end{aligned}
$$

with zero Dirichlet boundary conditions for both $T z$ and $T u$. Using Schauder's estimate on Eq. (3.4a), we can conclude that $T z \in C^{2+\gamma}(\bar{\Omega})$. Since $u \in S$, we have $u \leqslant w$. Hence taking the difference of the Eqs. (3.2) and (3.4a) gives $\Delta(\tilde{w}-T z)-\alpha(\tilde{w}-T z) \leqslant 0$ with zero boundary condition for $(\tilde{w}-T z)$. Hence the maximum principle gives $\tilde{w} \geqslant T z>0$ in $\Omega$. The strict inequality is a consequence of the strong maximum principle since $u \not \equiv 0$ in (3.4a). In addition, it follows from Lemma 1 that $\|T z\|_{C^{1}} \leqslant A$.

With $T z>0$ in $\Omega, u /(u+T z) \leqslant 1$. Hence $L^{p}$ theory guarantees a solution $T u \in W^{2, p}$ for Eq. (3.4b) with any $p>N$. In fact, since both $u$ and $T z$ are in $C^{1}(\bar{\Omega}), u /(u+T z)$ is in $C^{1}(\Omega)$. We can conclude that $T u \in C^{2}(\Omega)$ by interior Schauder's estimate, and can therefore employ the classical maximum principle on $T u$. Taking the difference of the Eqs. (3.1) and (3.4b) leads to $w \geqslant T u>0$ by using the (classical) maximum principle. In addition, lemma 1 gives $\|T u\|_{C^{1}} \leqslant A$.

Now for any $(u, z) \in S$, we have a uniform $L^{\infty}$ bound on $(1-\alpha) u$ and $u /(u+T z)$. This leads to a uniform $W^{2, p}$ norm bound on $T z$ and $T u$ for any $p>N$. Since $W^{2, p}$ is compactly contained in $C^{1}$, T is a compact map if we can show $T$ maps $S$ into $S$.

To show $T: S \rightarrow S$, it suffices to check the two integral inequalities in the definition of $S$. Everything else has been shown already. From (3.4a), multiply by $\varphi$ and integrate over $\Omega$,

$$
\left(\lambda_{1}+\alpha\right) \int_{\Omega} \varphi T z=(1-\alpha) \int_{\Omega} u \varphi \geqslant(1-\alpha) \delta .
$$

This ensures that $T z$ satisfies the second integral inequality in the definition of $S$.

Finally, we wish to verify $\int_{\Omega} \varphi T u \geqslant \delta$, dividing its proof into two cases. Define $\Omega_{\varepsilon} \equiv\{x \in \Omega$ : $\operatorname{dist}(x, \partial \Omega)>\varepsilon\}$. We will take $\varepsilon=\sqrt{\delta}$ in the following, with $\delta>0$ sufficiently small, to be determined in the proof.

Case I: $\delta \leqslant \int u \varphi \leqslant(1+M) \delta$.

In the following, all positive constants $m_{i}$ are independent of $\delta$. 
Since $w \in C^{1}(\bar{\Omega})$, for $x \in \Omega \backslash \Omega_{\sqrt{\delta}}$, we have $u(x) \leqslant w(x) \leqslant m_{1} \sqrt{\delta}$ for some constant $m_{1}>0$. Hence

$$
\int_{\Omega \backslash \Omega_{\sqrt{\delta}}} u \leqslant m_{1} \sqrt{\delta}\left|\Omega \backslash \Omega_{\sqrt{\delta}}\right| \leqslant m_{1} \sqrt{\delta}|\Omega| .
$$

Since the first eigenfunction $\varphi$ has non-zero slope at the boundary, there exists a positive constant $m_{2}$ such that

$$
\int_{\Omega_{\sqrt{\delta}}} u \leqslant\left(\int_{\Omega} u \varphi\right) /\left(\frac{\min }{\Omega_{\sqrt{\delta}}} \varphi\right) \leqslant \frac{(1+M) \delta}{m_{2} \sqrt{\delta}}=\frac{(1+M)}{m_{2}} \sqrt{\delta} .
$$

Adding the above two inequalities, $\|u\|_{1} \leqslant m_{3}(1+M) \sqrt{\delta}$ for some constant $m_{3}>0$, which is independent of $\delta$.

Since we have a uniform bound on the $C^{1}$ norm on $u \in S$, NirenbergGagliardo's inequality (statement (3) in Theorem 2.2, [10]) ensures that there exists a $\theta \in(0,1)$, and positive constants $m_{4}$ and $m_{5}$ such that for all $u \in S$,

$$
\|u\|_{\infty} \leqslant m_{4}\|u\|_{1}^{1-\theta} \leqslant m_{5}(1+M)^{1-\theta} \delta^{(1-\theta) / 2} \leqslant m_{5}(1+M) \delta^{(1-\theta) / 2} .
$$

Now from Eq. (3.4a), Lemma 1 gives $\|T z\|_{\infty} \leqslant k_{\alpha} m_{5}(1+M) \delta^{(1-\theta) / 2}$. Hence by choosing $\delta>0$ sufficiently small,

$$
1 /(u+T z) \geqslant 1 /\left[m_{5}(1+M)\left(1+k_{\alpha}\right) \delta^{(1-\theta) / 2}\right] \geqslant 2\left(1+\lambda_{1}\right) .
$$

Now, multiplying Eq. (3.4b) by $\varphi$ and integrating over $\Omega$, we have

$$
\left(1+\lambda_{1}\right) \int \varphi T u=\int \frac{u}{u+T z} \varphi \geqslant 2\left(1+\lambda_{1}\right) \int u \varphi \geqslant 2\left(1+\lambda_{1}\right) \delta .
$$

Therefore $\int \varphi T u \geqslant 2 \delta$. Hence $T: S \rightarrow S$ in this case.

Case II: $\int u \varphi \geqslant(1+M) \delta$.

From Eq. (3.4b),

$$
\begin{aligned}
\int \varphi T u & =\frac{1}{1+\lambda_{1}} \int \frac{u}{u+T z} \varphi \geqslant \frac{1}{\left(1+\lambda_{1}\right)\left(\|w\|_{\infty}+\|\tilde{w}\|_{\infty}\right)} \int u \varphi \\
& \geqslant \frac{1+M}{M} \delta \geqslant \delta .
\end{aligned}
$$

Hence $T$ maps $S$ into $S$ in both cases. By the Schauder's fixed point theorem, $T$ has a fixed point $(u, z)$, which satisfies Eqs. (3.3) with zero 
boundary conditions. Since $u \not \equiv 0$ in $\Omega$, we have $z>0$ in $\Omega$ by using the strong maximum principle on Eq. (3.3a). This in turn implies $u>0$ using Eq. (3.3b). Moreover, interior Schauder's estimate ensures that they are in $C^{2}(\Omega) \cap C^{1}(\bar{\Omega})$.

\section{EXISTENCE OF SOLUTIONS WHEN $\alpha>1$}

Define $g \in C^{2}(\bar{\Omega})$ to be the solution of

$$
\Delta g-\alpha g+1=0,\left.\quad g\right|_{\partial \Omega}=0 .
$$

First we need an existence result for a scalar singular equation.

LEMMA 2. - Let $\Omega$ be a bounded domain with $C^{2+\gamma}$ boundary. Assume $p \in C^{1}(\bar{\Omega}), p \geqslant 0$, and $\left.p\right|_{\partial \Omega}=0$. Then there exists a unique positive solution $u \in C^{2}(\Omega) \cap C^{1}(\bar{\Omega})$ to the singular equation

$$
\Delta u-\alpha u+\frac{p(x)}{u}+1=0,\left.\quad u\right|_{\partial \Omega}=0 .
$$

Moreover, $u \geqslant g$.

Proof. - We follow the proof in [9]. Essentially, although the equation is singular at the boundary, if we can construct a positive upper solution and positive lower solution with the upper solution larger than the lower solution, and both vanish at the boundary, then a classical solution in $C^{2}(\Omega) \cap C(\bar{\Omega})$ exists.

Define $\bar{u} \equiv A \sqrt{\varphi}$ for some constant $A>0$. Then

$$
\begin{aligned}
\Delta \bar{u} & -\alpha \bar{u}+\frac{p(x)}{\bar{u}}+1 \\
& =-\frac{\lambda_{1}}{2} A \sqrt{\varphi}-\frac{A}{4} \varphi^{-3 / 2}|\nabla \varphi|^{2}-\alpha A \sqrt{\varphi}+\frac{p(x)}{A \sqrt{\varphi}}+1 .
\end{aligned}
$$

Recall $\Omega_{\varepsilon} \equiv\{x \in \Omega$ : $\operatorname{dist}(x, \partial \Omega)>\varepsilon\}$. If $\varepsilon>0$ is sufficiently small, then there exists a constant $m>0$ such that $|\nabla \varphi|^{2} \geqslant m$ for all $x \in \bar{\Omega} \backslash \Omega_{\varepsilon}$. It is clear then

$$
\frac{A}{4} \varphi^{-3 / 2}|\nabla \varphi|^{2} \geqslant \frac{p(x)}{A \sqrt{\varphi}}+1,
$$

if we choose $A$ to be sufficiently large. 
For $x \in \overline{\Omega_{\varepsilon}}$, since there exists a $\delta>0$ such that $\min _{\overline{\Omega_{\varepsilon}}} \varphi \geqslant \delta$. By further increasing $A$ if necessary,

$$
\left(\frac{\lambda_{1}}{2}+\alpha\right) A \sqrt{\varphi} \geqslant \frac{p(x)}{A \sqrt{\varphi}}+1 .
$$

Thus $\Delta \bar{u}-\alpha \bar{u}+\frac{p(x)}{\bar{u}}+1 \leqslant 0$ for all $x \in \Omega$. Hence $\bar{u}$ is an an upper solution for Eq. (4.2) when $A$ is sufficiently large.

Next we define $\underline{u} \equiv \varepsilon_{0} \varphi$. By taking $\varepsilon_{0}>0$ sufficiently small, $\bar{u} \geqslant \underline{u}$. Reducing $\varepsilon_{0}$ further if necessary, we have

$$
\Delta \underline{u}-\alpha \underline{u}+\frac{p(x)}{\underline{u}}+1=-\lambda_{1} \varepsilon \varphi-\alpha \varepsilon_{0} \varphi+\frac{p(x)}{\varepsilon_{0} \varphi}+1>0 .
$$

Hence $\underline{u}$ is a lower solution, and there exists a classical solution $u \in$ $C^{2}(\Omega) \cap C(\bar{\Omega})$ for Eqs. (4.2). Uniqueness of such solution follows easily by using the maximum principle.

Now since $p \in C^{1}(\bar{\Omega})$ and $\left.p\right|_{\partial \Omega}=0$, we have $p \leqslant M \varphi$ for some constant $M>0$. Therefore,

$$
\frac{p(x)}{u} \leqslant \frac{M \varphi}{\varepsilon_{0} \varphi} \leqslant \frac{M}{\varepsilon_{0}} .
$$

This $L^{\infty}$ norm bound ensures that $u \in C^{1}(\bar{\Omega})$ by Lemma 1 . Finally the claim $u \geqslant g$ is a consequence of the maximum principle. This completes the proof of this lemma.

Analogous to the case when $\alpha<1$, we will study an equivalent system to Eqs. (2.2) when $\alpha>1$. This time we define $z \equiv u-v$, and study

$$
\begin{aligned}
\Delta z-z & =-(\alpha-1) v, \\
\Delta v-\alpha v+1+\frac{z}{v} & =0,
\end{aligned}
$$

with zero boundary conditions for both $z$ and $v$. Again we will employ Schauder's fixed point theorem in proving the following theorem.

THEOREM 4. - Let $\Omega$ be a bounded domain with $C^{2+\gamma}$ boundary. If $\alpha>1$, then there exist positive solutions $(u, v)$ in $C^{2}(\Omega) \cap C^{1}(\bar{\Omega})$.

Proof. - It suffices to establish the existence of positive solutions $(v, z)$ for Eqs. (4.3) with zero Dirichlet boundary conditions on both $v$ and $z$. Then $u=v+z$ will also be positive. 
Let $k_{1}$ be the constant in Lemma 1 when $\beta=1$, and $B \equiv 2 \sqrt{|\Omega|} \times$ $\max \{\alpha-1,1\}$. Let $A>1$, to be determined later, and $A_{1} \equiv k_{1}(\alpha-1) A$. Now define

$$
\begin{aligned}
S \equiv\left\{(v, z) \in C^{1}(\bar{\Omega}) \times C^{1}(\bar{\Omega}):\right. & v \geqslant g>0, z \geqslant 0,\left.v\right|_{\partial \Omega}=\left.z\right|_{\partial \Omega}=0, \\
& \left.\|v\|_{H^{1}} \leqslant B,\|v\|_{C^{1}} \leqslant A,\|z\|_{C^{1}} \leqslant A_{1}\right\} .
\end{aligned}
$$

For large $A, S$ is non-empty. Moreover, $S$ is closed, bounded, and convex.

Define the map $T$ such that for all $(v, z) \in S,(T v, T z)$ solve the system of equations:

$$
\begin{aligned}
\Delta T z-T z & =-(\alpha-1) v, \\
\Delta T v-\alpha T v+1+\frac{T z}{T v} & =0,
\end{aligned}
$$

with zero Dirichlet boundary conditions for both $T z$ and $T v$. Note that the first equation is linear. Using Schauder's estimate on Eq. (4.4a), we can conclude that $T z \in C^{2+\gamma}(\bar{\Omega})$. The maximum principle also gives $T z>0$. Moreover, it follows from Lemma 1 that

$$
\|T z\|_{C^{1}} \leqslant k_{1}\|(\alpha-1) v\|_{\infty} \leqslant k_{1}(\alpha-1) A=A_{1} .
$$

The second Eq. (4.4b) is a singular equation as in Lemma 2, and has a solution $T v \in C^{2}(\Omega) \cap C^{1}(\bar{\Omega})$, and $T v \geqslant g$.

If we can show $\|T v\|_{H^{1}} \leqslant B$, and $\|T v\|_{C^{1}} \leqslant A$, then $T: S \rightarrow S$. To prove the first inequality, multiplying Eq. (4.4a) by $T z$, and integrating over $\Omega$, we get $\|T z\|_{H^{1}} \leqslant(\alpha-1)\|v\|_{2}$ after using the Cauchy-Schwarz's inequality. Then from Eq. (4.4b), we have

$$
\begin{aligned}
\int_{\Omega}\left(|\nabla(T v)|^{2}+\alpha|T v|^{2}\right) & =\int_{\Omega}(T z+T v) \leqslant \sqrt{|\Omega|}\left(\|T z\|_{2}+\|T v\|_{2}\right) \\
& \leqslant \sqrt{|\Omega|}\left[(\alpha-1)\|v\|_{2}+\|T v\|_{2}\right] .
\end{aligned}
$$

Hence,

$$
\|T v\|_{H^{1}}^{2} \leqslant \frac{B}{2}\left(\|v\|_{2}+\|T v\|_{2}\right) \leqslant \frac{B}{2}\left(B+\|T v\|_{2}\right) .
$$

This quadratic inequality leads to:

$$
\|T v\|_{H^{1}} \leqslant B .
$$


This $H^{1}$ norm bound will help us to obtain the $C^{1}$ norm bound.

Let $m_{i}$ be some generic positive constants. Take any $x \in \Omega$. We have $g(x) \geqslant m_{1} \operatorname{dist}(x, \partial \Omega)$ for some $m_{1}>0$, and $T z(x)=|\nabla T z(\xi)| \operatorname{dist}(x$, $\partial \Omega$ ) by the mean value theorem for some $\xi \in \Omega$, which lies in the straight line connecting $x$ and its closest point to $\partial \Omega$. Hence fix some $p>N$,

$$
\frac{T z}{T v} \leqslant \frac{T z}{g} \leqslant \frac{1}{m_{1}}\|\nabla(T z)\|_{\infty} \leqslant \frac{m_{2}}{m_{1}}\|T z\|_{W^{2, p}} \leqslant m_{3}\|v\|_{p},
$$

by using the Sobolev's imbedding and $L^{p}$ estimate on Eq. (4.4a). Define $\theta \equiv(p-2) / p$. Hence $0<\theta<1$. We have $\|v\|_{p} \leqslant\|v\|_{\infty}^{\theta}\|v\|_{2}^{1-\theta}$. Combining with the inequality that we just obtained, we get

$$
\frac{T z}{T v} \leqslant m_{3} B^{1-\theta} A^{\theta} .
$$

Employing Lemma 1 on Eq. (4.4b) now with (4.6), and $A>1$, it follows that

$$
\|T v\|_{C^{1}} \leqslant k_{\alpha}\left\|1+\frac{T z}{T v}\right\|_{\infty} \leqslant k_{\alpha} \max \left\{1, m_{3} B^{1-\theta}\right\} 2 A^{\theta} \leqslant A,
$$

if we take $A$ sufficiently large in the last inequality. Hence $T: S \rightarrow S$.

Take $p>N$. For all $(v, z) \in S$, because of the bound (4.6), we have a $W^{2, p}$ norm bound on both $v$ and $z$. Since $W^{2, p}$ is compactly contained in $C^{1}(\Omega), T$ is a compact map.

By the Schauder's fixed point theorem, we have a fixed point $(v, z)$ in $S$ which satisfies Eqs. (4.3). The solution $v$ and $z$ are positive, and in $C^{2}(\Omega) \cap C^{1}(\bar{\Omega})$ by similar arguments as in the proof of Theorem 3 .

Theorems 2 to 4 establish the existence of solution as summarized in Theorem 1. We now move on to study uniqueness of solution for one dimensional domains in the next section.

\section{UNIQUENESS OF THE SOLUTION IN ONE DIMENSION}

Without loss of generality, we let the domain $\Omega$ be $(0,1)$, and study the uniqueness of positive solutions $(u, v)$ in $C^{2}(0,1) \cap C^{1}[0,1]$ satisfying Eqs. (2.2). There is no distinction between the $\alpha<1$ case and the $\alpha>1$ case.

Because $u / v>0$ in $\Omega$, then from Eq. (2.2a), we have $u^{\prime \prime}-u<0$ in $(0,1)$. Application of the Hopf's lemma (p. 5, Theorem 2, [14]) gives 
$u^{\prime}(0)>0$. Similarly, Eq. (2.2b) yields $v^{\prime}(0)>0$. Similar analysis can be made at $x=1$. Now as $x \rightarrow 0$, we have

$$
\frac{u}{v} \rightarrow \frac{u^{\prime}(0)}{v^{\prime}(0)} .
$$

Thus all solutions $(u, v)$ in $C^{2}(0,1) \cap C^{1}[0,1]$ are in fact $C^{2}[0,1]$. We need an extension of the result in Lemma 2.

Lemma 3. - Let the same assumptions in Lemma 2 hold. Let $u_{i}$ be the positive solution of Eqs. (4.2) with zero boundary conditions when $p(x)$ is $p_{i}(x), i=1,2$. Assume $p_{2}(x) \geqslant p_{1}(x)$ in $[0,1]$ and $p_{2} \not \equiv p_{1}$, then $u_{2}>u_{1}$ in $[0,1]$.

Proof. - Define $\hat{u} \equiv u_{2}-u_{1}$. Then from Eq. (4.2) for $i=1,2$, we obtain

$$
\hat{u}^{\prime \prime}-\alpha \hat{u}-\frac{p_{2}(x)}{u_{1} u_{2}} \hat{u}=-\frac{p_{2}(x)-p_{1}(x)}{u_{1}} \leqslant 0 .
$$

Since $\left.p_{2}\right|_{\partial \Omega}=0$, and $u_{i}^{\prime}(0)>0$ for $i=1,2$, hence the positive coefficient $p_{2}(x) /\left(u_{1} u_{2}\right)$ behaves like $1 / x$ as $x \rightarrow 0$. Now an improved version of the maximum principle (p. 6, Theorem 3, [14]) allows for such unbounded coefficient (so long as we have a sign restriction), we can therefore conclude $\hat{u}>0$ in $[0,1]$ unless $\hat{u}$ is identically zero. Since $p_{2} \not \equiv p_{1}$ in $[0,1]$, we can exclude this case. The proof of this lemma is now complete.

LEMMA 4. - Let $\left(u_{i}, v_{i}\right) \in C^{2}[0,1]$ be positive solutions of (2.2) with zero boundary conditions, $i=1,2$. Assume $\left(u_{2}, v_{2}\right) \not \equiv\left(u_{1}, v_{1}\right)$ in $[0,1]$. Then $u_{2} \not \equiv u_{1}$ and $v_{2} \not \equiv v_{1}$.

Proof. - Note that if $u_{2} \equiv u_{1}$, then $v_{2} \equiv v_{1}$ from the governing Eq. (2.2a). Similarly when $v_{2} \equiv v_{1}$, then $u_{2} \equiv u_{1}$ by using Eq. (2.2b). Since $\left(u_{2}, v_{2}\right) \not \equiv\left(u_{1}, v_{1}\right)$ in $[0,1]$, we have $u_{2} \not \equiv u_{1}$ and $v_{2} \not \equiv v_{1}$.

THEOREM 5. - Let $\left(u_{i}, v_{i}\right) \in C^{2}[0,1]$ be positive solutions of (2.2) with zero boundary conditions, $i=1,2$. Assume $\left(u_{2}, v_{2}\right) \not \equiv\left(u_{1}, v_{1}\right)$ in $[0,1]$. Then $u_{2} ¥ u_{1}$ and $v_{2} ¥ v_{1}$, i.e., neither component of each solution can be ordered.

Proof. - We will assume $v_{2} \geqslant v_{1}$ in $[0,1]$, and arrive at a contradiction. First we note that

$$
u_{i}^{\prime \prime}+\left(\frac{1}{v_{i}}-1\right) u_{i}=0, \quad i=1,2 .
$$


Multiplying the first equation by $u_{2}$, the second equation by $u_{1}$, and subtracting one equation from another, we have

$$
\int_{0}^{1}\left(\frac{1}{v_{2}}-\frac{1}{v_{1}}\right) u_{1} u_{2}=0 .
$$

Since $v_{2} \not \equiv v_{1}$ by Lemma 4 , this gives a contradiction. Hence $v_{2} \ngtr v_{1}$.

Next we assume $u_{2} \geqslant u_{1}$. From Lemmas 3 and 4 , we obtain $v_{2}>v_{1}$. We have just proved that this is impossible. Hence this contradiction enables us to conclude $u_{2} \ngtr u_{1}$.

Suppose there are two distinct positive solutions $\left(u_{i}, v_{i}\right), i=1,2$. Define $\hat{u} \equiv u_{2}-u_{1}$ and $\hat{v} \equiv v_{2}-v_{1}$. In the above theorem, we have just established that neither $\hat{u}$ nor $\hat{v}$ can be single-signed. We now proceed to exclude the sign changing cases for $\hat{u}$ and $\hat{v}$.

We will need the following lemma, the proof of which we postpone till the next section.

LEMMA 5. - Both $\hat{u}$ and $\hat{v}$ have finite number of zeros in $[0,1]$, unless they are identically zero.

We now prove the uniqueness of solution under this additional assumption. Define

$$
\begin{aligned}
& \hat{I_{+}} \equiv\{x \in[0,1]: \hat{u} \geqslant 0\}, \\
& \hat{I_{-}} \equiv\{x \in[0,1]: \hat{u} \leqslant 0\}, \\
& \hat{J}_{+} \equiv\{x \in[0,1]: \hat{v} \geqslant 0\}, \\
& \hat{J}_{-} \equiv\{x \in[0,1]: \hat{v} \leqslant 0\} .
\end{aligned}
$$

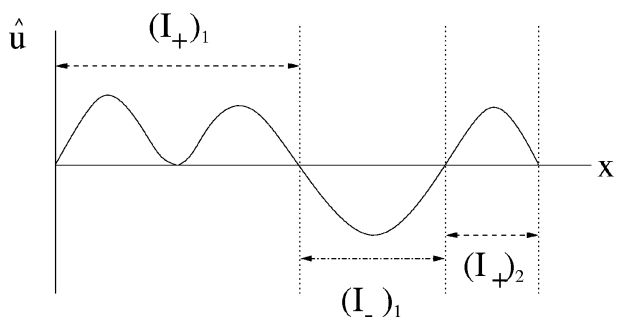

Fig. 1. Definition of $I_{+}$. 
Because of the Lemma $5, \hat{I_{+}}$consists of finitely many disjoint closed intervals, say $\left(I_{+}\right)_{i}, i=1, \ldots, m_{1}$, i.e., $\hat{I_{+}}=\bigcup_{i}\left(I_{+}\right)_{i}$. It is noted that in each $\left(I_{+}\right)_{i}$, there can be at most finitely many points at which $\hat{u}=0$. To simplify our notation, we employ $I_{+}$to denote any interval $\left(I_{+}\right)_{i}$. Similar remarks apply for $I_{-}, J_{+}$, and $J_{-}$(see Fig. 1).

LEMMA 6. - The following four cases hold:

(a) $I_{+} \not \subset J_{+}$,

(b) $I_{-} \not \subset J_{-}$,

(c) $J_{-} \not \subset I_{+}$,

(d) $J_{+} \not \subset I_{-}$.

Proof. - Examine case (a) first. Assume $I_{+} \subset J_{+}$, and let $I_{+}=[a, b]$ for some $0 \leqslant a<b \leqslant 1$. From both Eqs. (5.1) for $i=1,2$, simple algebraic manipulation yields

$$
\left.\left(u_{1} u_{2}^{\prime}-u_{2} u_{1}^{\prime}\right)\right|_{x=a} ^{x=b}+\int_{a}^{b}\left(\frac{1}{v_{2}}-\frac{1}{v_{1}}\right) u_{1} u_{2}=0 .
$$

Since $u_{1}(a)=u_{2}(a)$ and $u_{1}(b)=u_{2}(b)$, the above equation is simplified to

$$
\begin{array}{r}
\left\{u_{1}(b)\left(u_{2}^{\prime}(b)-u_{1}^{\prime}(b)\right)-u_{1}(a)\left(u_{2}^{\prime}(a)-u_{1}^{\prime}(a)\right)\right\} \\
+\int_{a}^{b}\left(\frac{1}{v_{2}}-\frac{1}{v_{1}}\right) u_{1} u_{2}=0 .
\end{array}
$$

Since $u_{2}^{\prime}(b) \leqslant u_{1}^{\prime}(b)$ and $u_{2}^{\prime}(a) \geqslant u_{2}^{\prime}(a)$, the first two terms on the left hand side are non-positive. The integral term is strictly negative, since $[a, b] \subset J_{+}$, and $\hat{v}$ is zero only on finitely many points on $(a, b)$. Hence we arrive at a contradiction. The proof of case (a) is complete. The proof of case (b) is similar by studying the same Eqs. (5.1).

Next we look at case (c). Assume $J_{-} \subset I_{+}$and let $J_{-}=[a, b]$ for some $0 \leqslant a<b \leqslant 1$. It is clear that $u_{2} / v_{2}>u_{1} / v_{1}$ on $(a, b)$. Hence from Eqs. (2.2) for $i=1,2$, we get

$$
\hat{v}^{\prime \prime}-\alpha \hat{v}=-\left(\frac{u_{2}}{v_{2}}-\frac{u_{1}}{v_{1}}\right)<0,
$$

with the boundary conditions $\hat{v}(a)=\hat{v}(b)=0$. The maximum principle gives $\hat{v}>0$ on $(a, b)$. This contradicts the interval $[a, b]=J_{-}$. The proof 
of case (c) is complete. The proof of case (d) is similar to that for case (c).

THEOREM 6. - Let $\Omega$ be a bounded interval in the one dimensional case. Then positive solutions $(u, v)$ in $C^{2}(0,1) \cap C^{1}[0,1]$ are unique.

Proof. - Without loss of generality, by picking which solution we assign as $i=1$, we can assume $\hat{u}>0$ in a neighborhood of $x=0$. Let $\hat{u}>0$ on $\left[0, a_{1}\right],\left[a_{2}, a_{3}\right], \ldots,\left[a_{2 n}, a_{2 n+1}\right]$, and $\hat{u}<0$ on $\left[a_{1}, a_{2}\right],\left[a_{3}, a_{4}\right]$, $\ldots$ Thus $a_{2 n+1}=1$, or $a_{2 n+2}=1$, depending on whether the last interval that $\hat{u}$ does not vanish is positive or negative.

We divide into two cases in studying the interval $\left[0, a_{1}\right]$.

Case I: $\hat{v}>0$ near $x=0$.

On the interval $\left(0, a_{1}\right), \hat{v}$ has to change sign exactly once. For if $\hat{v}$ does not change sign, then it contradicts case (a) in Lemma 6. If $\hat{v}$ changes sign more than once, then it contradicts case (c) in Lemma 6.

Case II: $\hat{v}<0$ near $x=0$.

On the interval $\left(0, a_{1}\right), \hat{v}$ cannot change sign. For if $\hat{v}$ changes sign, then it contradicts case (c) in Lemma 6.

Hence in both cases, $\hat{v}$ is (strictly) negative at $x=a_{1}$. It has to change sign exactly once inside the interval $\left(a_{1}, a_{2}\right)$. For if $\hat{v}$ does not change sign in $\left(a_{1}, a_{2}\right)$, it contradicts case (b) in Lemma 6 . If $\hat{v}$ changes sign more than once, then it contradicts case (d) in Lemma 6. Thus $\hat{v}>0$ at $x=a_{2}$.

A repetition of such arguments gives $\hat{v}<0$ at $x=a_{3}, \hat{v}>0$ at $x=a_{4}$, and so on. If the last interval is $\left[a_{2 n}, 1\right]$, then we know $\hat{v}>0$ at $x=a_{2 n}$. Because of case (a) in Lemma $6, \hat{v}$ has to change sign at some interior point in $\left(a_{2 n}, a_{2 n+1}\right)$. But then $\hat{v}$ has to become zero at or before $x=1$ because of its boundary condition. It will contradict case (c) in Lemma 6 .

The case that the last interval is $\left[a_{2 n+1}, 1\right]$ where $\hat{u}<0$ can be treated similarly. This complete the uniqueness proof if Lemma 5 holds.

The proof of Lemma 5 is the contents of the next section.

\section{PROOF OF LEMMA 5}

In this section, we will establish Lemma 5. This will finish the proof of Theorem 1 as well. 
LEMMA 7. - Let $\mathbf{w} \equiv\left(w_{1}, w_{2}\right) \in C^{2}[a, b]$ be a nontrivial solution of a linear system of two equations on the interval $[a, b]$ :

$$
\mathbf{w}^{\prime \prime}+A(x) \mathbf{w}=0,
$$

where $A \in C[a, b]$. If $A_{12}(x) \neq 0$ and $A_{21}(x) \neq 0$ for all $x \in[a, b]$, then neither $w_{1}$ nor $w_{2}$ can have infinite number of zeros in $[a, b]$.

Proof. - First we assume that there are infinite number of zeros $\left\{x_{n}\right\}$, $n=1,2, \ldots$, for $w_{1}$ in $[a, b]$. Take a subsequence of $\left\{x_{n}\right\}$ if necessary, we can assume $x_{n} \rightarrow x_{0}$ for some $x_{0} \in[a, b]$. Without loss of generality, assume that $\left\{x_{n}\right\}$ is a monotone decreasing sequence. (The other case that it is a monotone increasing sequence can be treated in the same way.)

By Rolle's theorem, we can readily conclude that $w_{1}\left(x_{0}\right)=0, w_{1}^{\prime}\left(x_{0}\right)$ $=0$, and $w_{1}^{\prime \prime}\left(x_{0}\right)=0$. Evaluate Eq. (6.1a) at $x=x_{0}$, we have $A_{12}\left(x_{0}\right) w_{2}$ $\left(x_{0}\right)=0$. Since by assumption $A_{12}\left(x_{0}\right) \neq 0$, we obtain $w_{2}\left(x_{0}\right)=0$. If $w_{2}^{\prime}\left(x_{0}\right)=0$, then uniqueness of initial value problem for Eqs. (6.1) forces $w_{1} \equiv 0$ and $w_{2} \equiv 0$ for all $x \in[a, b]$. Since $\mathbf{w}$ is not a trivial solution, we can assume that $w_{2}^{\prime}\left(x_{0}\right) \neq 0$.

Let $\delta>0$ be sufficiently small and $x \in\left(x_{0}, x_{0}+\delta\right]$. Then

$$
\left|A_{12}(x) w_{2}(x)\right|>\left|A_{11}(x) w_{1}(x)\right|,
$$

because $w_{1}(x)$ behaves like $\mathrm{o}\left(\left(x-x_{0}\right)^{2}\right),\left|w_{2}(x)\right| \geqslant m\left(x-x_{0}\right)$ for some $m>0$, and $\left|A_{12}(x)\right|$ has a positive minimum on the interval $[a, b]$. Hence Eq. (6.1a) gives $w_{1}^{\prime \prime}$ is non-zero and is single-signed for $x \in\left(x_{0}, x_{0}+\delta\right]$. This contradicts that there are infinite zeros of $w_{1}$ on $\left(x_{0}, x_{0}+\delta\right]$. Hence $w_{1}$ has to have finite number of zeros in $[a, b]$.

The proof of $w_{2}$ having finite number of zeros in $[a, b]$ is similar. It invokes the assumption $A_{21}$ is nonzero for all $x \in[a, b]$.

From Eqs. (2.2), a simple calculation shows that if $\mathbf{w} \equiv(\hat{u}, \hat{v})$, then $\mathbf{w}$ satisfies Eqs. (6.1) with matrix

$$
A=\left(\begin{array}{cc}
\frac{1}{v_{2}}-1 & -\frac{u_{1}}{v_{1} v_{2}} \\
\frac{1}{v_{2}} & -\frac{u_{1}}{v_{1} v_{2}}-\alpha
\end{array}\right) .
$$

This matrix $A$ is singular near the boundary $x=0$ and $x=1$. However for any subinterval $[a, b] \subset(0,1)$, Lemma 7 allows us to conclude that there are finite number of zeros in $[a, b]$ for $\hat{u}$ and $\hat{v}$. It remains to show 
that there are no infinite number of zeros of $\hat{u}$ and $\hat{v}$ near $x=0$ and $x=1$. First we recall the following simple theorem.

THEOREM 7. - If $f \in C[0,1]$, and $u \in C^{2}[0,1]$ satisfies $u^{\prime \prime}=f(x)$ with $u(0)=u^{\prime}(0)=0$, then for any $x \in[0,1]$,

$$
u(x)=\int_{0}^{x}(x-t) f(t) \mathrm{d} t .
$$

We need this formula to establish the uniqueness theorem of initial value problem for a singular, linear system of equations below.

LEMMA 8. - Let Eqs. (6.1) hold with $\mathbf{w} \in C^{2}[0,1 / 2]$. Moreover $\mathbf{w}(0)=0, \mathbf{w}^{\prime}(0)=0$, and $A \in C(0,1 / 2]$. Furthermore, let $\lim _{x \rightarrow 0} x A(x)$ exist. Then $\mathbf{w} \equiv 0$ on $[0,1 / 2]$.

Proof. - Define the matrix $B(x) \equiv x A(x)$. Hence $B \in C[0,1 / 2]$ due to the assumption on the existence of the above limit. Let $M \equiv$ $\max _{0 \leqslant x \leqslant 1 / 2}\|B(x)\|_{\infty}$ (where the infinity norm here is the infinity matrix norm on a $2 \times 2$ matrix), and $k \equiv \max _{0 \leqslant x \leqslant 1 / 2}\left\|\frac{1}{x} \mathbf{w}(x)\right\|_{\infty}$ (where the infinity norm here is the infinity vector norm on a $2 \times 1$ vector). It is noted that $k$ is well defined because $\mathbf{w}(0)=0$, and $\mathbf{w}$ is in $C^{1}[0,1 / 2]$.

Now formula (6.3) can be applied to yield

$$
\mathbf{w}(x)=\int_{0}^{x}(x-t) B(t) \frac{\mathbf{w}}{t} \mathrm{~d} t,
$$

which in turns lead to:

$$
\|\mathbf{w}\|_{\infty} \leqslant \int_{0}^{x}(x-t)\|B(t)\|_{\infty}\left\|\frac{\mathbf{w}}{t}\right\|_{\infty} \mathrm{d} t .
$$

Hence,

$$
\|\mathbf{w}\|_{\infty} \leqslant M k \int_{0}^{x}(x-t) \mathrm{d} t=\frac{M k}{2} t^{2} .
$$

Putting Eq. (6.5) back into Eq. (6.4), we obtain an improved estimate:

$$
\|\mathbf{w}\|_{\infty} \leqslant \frac{M k^{2}}{12} t^{3} \leqslant \frac{M k^{2}}{3 !} t^{3} .
$$


Using induction, it is easy to check that for any positive integer $n$,

$$
\|\mathbf{w}\|_{\infty} \leqslant \frac{M k^{n}}{(n+1) !} t^{n+1} .
$$

Hence $\|\mathbf{w}\|_{\infty} \leqslant M k^{n} /\left(2^{n+1}(n+1) !\right)$. Take $n \rightarrow \infty,\|\mathbf{w}\|_{\infty} \leqslant 0$. Hence $\mathbf{w} \equiv 0$ on $[0,1 / 2]$.

We now finish the proof of Lemma 5 by showing that no infinite number of zeros of $\hat{u}$ and $\hat{v}$ near $x=0$ and $x=1$. We focus on $x=0$. The other end $x=1$ is similar. We first consider three cases.

Case I: $\hat{u}$ has infinite zeros in a neighborhood of $x=0$, and $\hat{v}^{\prime}(0) \neq 0$.

Because of the existence of infinite zeros of $\hat{u}$ near $x=0$, we have $\hat{u}^{\prime}(0)=0$. Thus $u_{1}^{\prime}(0)=u_{2}^{\prime}(0)$. With $\hat{v}^{\prime}(0) \neq 0$, we know $v_{1}^{\prime}(0) \neq v_{2}^{\prime}(0)$. (Recall that both $v_{1}^{\prime}(0)$ and $v_{2}^{\prime}(0)$ are positive.)

Now from Eq. (2.2a) for $i=1,2$, we can derive:

$$
\hat{u}^{\prime \prime}=\hat{u}-\left(\frac{u_{2}}{v_{2}}-\frac{u_{1}}{v_{1}}\right) .
$$

Since

$$
\frac{u_{i}}{v_{i}} \rightarrow \frac{u_{i}^{\prime}(0)}{v_{i}^{\prime}(0)} \quad \text { for } i=1,2,
$$

hence $\left|u_{2} / v_{2}-u_{1} / v_{1}\right|$ is bounded away from zero in a sufficiently small neighborhood $[0, \delta]$ for some $\delta>0$. Reduce $\delta$ further if necessary, the right-hand side of Eq. (6.7) is single-signed and non-zero in $[0, \delta]$, because $\hat{u}(0)=0$. Since $\hat{u}(0)=0$ and $\hat{u}^{\prime}(0)=0$, this will contradict that $\hat{u}$ has infinite number of zero in $[0, \delta]$. Hence case I cannot exist.

Case II: $\hat{u}$ has infinite zeros in a neighborhood of $x=0$, and $\hat{v}^{\prime}(0)=0$.

In this case, $\hat{u}(0)=\hat{u}^{\prime}(0)=0$ and $\hat{v}(0)=\hat{v}^{\prime}(0)=0$. Since $\mathbf{w} \equiv(\hat{u}, \hat{v})$ satisfies Eqs. (6.1) with the matrix $A$ given by (6.2), Lemma 8 gives $\hat{u} \equiv 0$ and $\hat{v} \equiv 0$ on the interval $[0,1 / 2]$. Now we can use Lemma 7 to conclude that they are identically zero on $[0,1]$. Hence case II is equivalent to identically zero $\hat{u}$ and $\hat{v}$.

Case III: $\hat{v}$ has infinite zeros in a neighborhood of $x=0$, and $\hat{u}^{\prime}(0) \neq 0$.

Using similar proof as in case I on Eq. (2.2b), we have

$$
\hat{v}^{\prime \prime}=\alpha \hat{v}-\left(\frac{u_{2}}{v_{2}}-\frac{u_{1}}{v_{1}}\right) .
$$

The same argument shows that this case does not exist. 
When there are infinite number of zeros for $\hat{u}$ near $x=0$, and only finite number of zeros for $\hat{v}$, this is either case I or case II. But case I does not exist. Thus from the conclusion of case II, $\hat{u} \equiv 0$ and $\hat{v} \equiv 0$ on $[0,1]$.

Similar remark applies when there are infinite number of zeros for $\hat{v}$ near $x=0$ and only finite number zeros for $\hat{u}$. Since case III does not exist, we end up with case II again.

When there are infinite number of zeros for both $\hat{u}$ and $\hat{v}$ near $x=0$, this is case II. Hence we draw the same conclusion that both $\hat{u}$ and $\hat{v}$ are identically zero on $[0,1]$. The proof of Lemma 5 is now complete.

\section{CONCLUDING REMARKS ... WHAT'S LEFT}

We conclude this paper with a short outline of the main open questions left by this paper.

To some extent, in taking all the exponents in the coupling terms of (1.1) to be one, we have made the equation less singular than it otherwise would be. The most obvious open question is what happens for different exponents. Presumably, for existence, we need a critical exponent limitation on the $p$ and $r$, and (if the single-equation literature is any guide) no restriction on the $q$ and $s$. To our knowledge, there are no additional results even in the one-dimensional case or when radial symmetry is imposed.

In addition, we have no information on whether the solutions we find are radially symmetry if the region is a ball. It is natural to ask if all positive solutions of (2.2) are radially symmetric or if some symmetrybreaking can result.

A stronger version of this question is whether the positive-positive solution is always unique. Note that uniqueness is still open even in one dimension for the more general exponent case. For other values of $p, q$, $r, s$, formal arguments on Eqs. (1.1) leads to a single elliptic equation, which has multiple solutions for Neumann boundary conditions.

And finally, one can wonder whether these methods can be generalized to a wider class of nonlinearities than the pure exponents.

\section{REFERENCES}

[1] C̆anić S., Lee Keyfitz B., An elliptic problem arising form the unsteady transonic small disturbance equation, J. Differential Equations 125 (1996) 548-574.

[2] Choi Y.S., Kim E.H., On the existence of positive solutions of quasilinear elliptic boundary value problems, J. Differential Equations 155 (1999) 423-442. 
[3] Choi Y.S., Lazer A.C., McKenna P.J., On a singular quasilinear anisotropic elliptic boundary value problem, Trans. Amer. Math. Soc. 347 (1995) 2633-2641.

[4] Choi Y.S., McKenna P.J., On a singular quasilinear anisotropic elliptic boundary value problem, II, Trans. Amer. Math. Soc. 350 (1998) 2925-2937.

[5] Crandall M.G., Rabinowitz P.H., Tartar L., On a Dirichlet problem with a singular nonlinearity, Comm. PDE 2 (2) (1997) 193-222.

[6] del Pino M.A., Radially symmetric layers in a semilinear elliptic system, Trans. Amer. Math. Soc. 347 (1995) 4807-4837.

[7] Gui C., Multipeak solutions for a semilinear Neumann problem, Duke Math. J. 84 (1996) 739-769.

[8] Gilbarg D., Trudinger N.S., Elliptic Partial Differential Equations of Second Order, 2nd ed., Springer, 1983.

[9] Lazer A.C., McKenna P.J., On a singular nonlinear elliptic boundary value problem, Proc. Amer. Math. Soc. 111 (1991) 721-730.

[10] Ladyzenskaja O.A., Solonnikov V.A., Ural'ceva N.N., Linear and Quasilinear Equations of Parabolic Type, Translation of Mathematical Monographs, Vol. 23, MS, 1968.

[11] Nachman A., Callegari A., A nonlinear singular boundary value problem in the theory of pseudoplastic fluids, SIAM J. Appl. Math. 28 (5) (1996) 271-281.

[12] Ni W.-M., Takagi I., On the location and profile of spike-layer solutions to a singularly perturbed semilinear Dirichlet problem: intermediate solutions, Duke Math. J. 94 (1998) 597-618.

[13] Ni W.-M., Diffusion, cross-diffusion, and their spike-layer steady states, Notices Amer. Math. Soc. 45 (1998) 9-18.

[14] Protter M., Weinberger H., Maximum Principles in Differential Equations, PrenticeHall, Englewood Cliffs, NJ, 1984.

[15] Reichel W., Uniqueness for degenerate elliptic equations via Serrin's sweeping principle, General Inequalities 7, International Series of Numerical Mathematics, Birkhauser, Basel (to appear).

[16] Sattinger D.H., Topics in Stability and Bifurcation Theory, Lecture Notes in Mathematics, Vol. 309, Springer-Verlag, 1973.

[17] Serrin J., Zou H., Existence of positive entire solutions of elliptic Hamiltonian systems, Comm. Partial Differential Equations 23 (1998) 577-599.

[18] Lair A., Wood A., Existence of entire solutions of semilinear elliptic systems, Preprint.

[19] Stuart C.A., Existence theorems for a class of nonlinear integral equations, Math. Z. 137 (1974) 49-66.

[20] Taliaferro S.D., A nonlinear singular boundary value problem, Nonlinear Analysis TMA 3 (1979) 897-904.

[21] Turchin P., Reeve J.D., Cronin J.T., Spatial pattern formation in ecological systems: bridging theoretical and empirical approaches, in: Bascompte J., Sole R.V. (Eds.), Modelling Spatiotemporal Dynamics in Ecology, Pitman, 1997, pp. 195-210. 